\title{
Partir pour la ville : étude des caractéristiques des pensionnaires dans la ville de Québec au tournant $\mathrm{du}_{\mathrm{XX}} \mathrm{e}^{\mathrm{e}}$ siècle
}

\section{Valérie Laflamme}

Volume 55, numéro 3, hiver 2002

URI : https://id.erudit.org/iderudit/010416ar

DOI : https://doi.org/10.7202/010416ar

Aller au sommaire du numéro

Éditeur(s)

Institut d'histoire de l'Amérique française

ISSN

0035-2357 (imprimé)

1492-1383 (numérique)

Découvrir la revue

Citer cet article

Laflamme, V. (2002). Partir pour la ville : étude des caractéristiques des pensionnaires dans la ville de Québec au tournant du $\mathrm{XX}^{\mathrm{e}}$ siècle. Revue d'histoire de l'Amérique française, 55(3), 407-425.

https://doi.org/10.7202/010416ar

\section{Résumé de l'article}

Le présent travail, qui se veut exploratoire, cherche à faire sortir de l'ombre le groupe des personnes qui paient pension à un particulier ou à un établissement de pension pour que leur soient fournis nourriture et logement, ceux qu'on appelle les pensionnaires. À partir des données nominatives du recensement canadien de 1901 pour la ville de Québec, nous tentons de dégager certaines des caractéristiques sociodémographiques de ces pensionnaires. Cette analyse met en évidence l'hétérogénéité de la population des pensionnaires. Il en ressort qu'être pensionnaire est une pratique sexuée et liée à l'âge. Les pensionnaires sont originaires des régions rurales aussi bien qu'urbaines et rien n'indique une surreprésentation d'une communauté ethnoreligieuse particulière. Enfin, leur répartition socioprofessionnelle souligne à nouveau l'hétérogénéité de la population des pensionnaires.
Tous droits réservés @ Institut d'histoire de l'Amérique française, 2000
Ce document est protégé par la loi sur le droit d'auteur. L'utilisation des services d’Érudit (y compris la reproduction) est assujettie à sa politique d'utilisation que vous pouvez consulter en ligne.

https://apropos.erudit.org/fr/usagers/politique-dutilisation/ 


\title{
Partir pour la ville: étude des caractéristiques des pensionnaires dans la ville de Québec au tournant du $\mathrm{xx}^{\mathrm{e}}$ siècle $^{\mathrm{I}}$
}

\author{
VALÉRIE LAFLAMME \\ École des hautes études en sciences sociales \\ Institut national d'études démographiques
}

RÉSUMÉ - Le présent travail, qui se veut exploratoire, cherche à faire sortir de l'ombre le groupe des personnes qui paient pension à un particulier ou à un établissement de pension pour que leur soient fournis nourriture et logement, ceux qu'on appelle les pensionnaires. $\grave{A}$ partir des données nominatives du recensement canadien de I90I pour la ville de Québec, nous tentons de dégager certaines des caractéristiques sociodémographiques de ces pensionnaires. Cette analyse met en évidence l'hétérogénéité de la population des pensionnaires. II en ressort qu'être pensionnaire est une pratique sexuée et liée à l'âge. Les pensionnaires sont originaires des régions rurales aussi bien qu'urbaines et rien n'indique une surreprésentation d'une communauté ethnoreligieuse particulière. Enfin, leur répartition socioprofessionnelle souligne à nouveau l'hétérogénéité de la population des pensionnaires.

ABSTRACT - This exploratory note seeks to a better understanding of the features of the specific group of individuals formed by boarders. Through a quantitative analysis of the nominative data given by the 190 I Canadian Census for the City of Quebec, we study the prevailing socio-demographical characteristics of the boarders. The analysis shows the absence of homogeneity within this group. Being a boarder has gender orientations. It also varies according to the age. Half of the boarders are born in rural areas. Nothing indicates an over-representation of any ethnic or religious group. Finally, the heterogeneity of the group formed by boarders is shown again by an analysis of their occupations.

1. Cette note de recherche fut présentée au colloque La famille et les transformations économiques, $X V I I I^{e}-X X^{e}$ siècles tenu à Montréal en novembre 2001. Je remercie Alain Blum, Catherine Bonvalet et Christian Dessureault pour leurs conseils, ainsi que les évaluateurs de la Revue. Les données exploitées ont été saisies dans le cadre du programme de recherche Population et histoire sociale de la ville de Québec (PHSVQ) du CIEQ. Nous remercions les codirecteurs, Richard Marcoux et Marc St-Hilaire, ainsi que Charles Fleury pour la mise en forme de la base de données. Mes recherches reçoivent l'appui du CRSH, du FCAR et de l'Institut national d'études démographiques. 
u tournant du xx ${ }^{\mathrm{e}}$ siècle, la ville de Québec était, pour reprendre
l'expression de Raoul Blanchard, un «foyer d'appel» pour de nombreux ruraux de l'est de la province ${ }^{2}$. Celle-ci connaît à cette époque de forts mouvements de population : les résidants des campagnes se déplacent en grand nombre vers la ville, soit temporairement pour y travailler, soit définitivement pour s'y installer. Ces mouvements de population à l'intérieur de la province sont encore peu connus ${ }^{3}$, il semble cependant que ces migrations vers la ville revêtaient un caractère provisoire et transitoire, et que la ville de Québec pouvait à la fois constituer un point d'arrêt, une halte ou un tremplin vers d'autres destinations ${ }^{4}$. Nous pouvons donc supposer que l'arrivée en ville des ruraux prenait fréquemment la forme d'un hébergement temporaire, comme celui de la pension.

Rappelons qu'à la suite de transformations économiques et politiques, la population de la ville de Québec s'est renouvelée à plus de $80 \%$ en 3 décennies $^{5}$. Cette modification du tissu social de la ville peut aussi avoir favorisé le développement de formes de «logements de passage ${ }^{6}$ », à caractère transitoire ou provisoire, destinés à l'intégration des nouveaux arrivants.

Pensons à Anna Pettigrew, une modiste de 26 ans qui, au moment où les agents recenseurs sillonnent les rues de Québec en 1901, loge dans le quartier Saint-Roch chez Michel Consigny et sa famille ${ }^{7}$. Ou encore à Adélard et Odilon Lamothe, deux frères âgés de 18 et 20 ans, qui travaillent l'un comme corroyeur et l'autre comme boulanger et prennent pension à Saint-Sauveur chez les Gagnon ${ }^{8}$. Il y a aussi Eugène Ouimet, 33 ans, et John Burns, 28 ans,

2. Raoul Blanchard, L'est du Canada français, province de Québec (Paris, Montréal, 1935), 239.

3. Voir notamment Yolande Lavoie, «Les mouvements migratoires des Canadiens entre leur pays et les États-Unis au $\mathrm{XIx}^{\mathrm{e}}$ et au $\mathrm{Xx}^{\mathrm{e}}$ siècles : étude quantitative », dans Hubert Charbonneau, dir., La population du Québec : études rétrospectives (Montréal, Boréal Express, 1973), 73-88.

4. Bruno Ramirez, Crossing the $49^{\text {th }}$ Parallel : Migration to the United States, 1900-1930 (Ithaca, Cornell University Press, 2001), 140.

5. Cette différence est encore plus marquée selon les trois principaux groupes ethniques : au contraire des Irlandais catholiques et des Britanniques protestants pour lesquels les soldes migratoires sont négatifs, le solde migratoire des Canadiens français demeure presque nul, puisque chaque départ se voit compensé par une arrivée. Marc St-Hilaire et Richard Marcoux, «Le ralentissement démographique», dans Serge Courville et Robert Garon, dir., Québec, ville et capitale (Sainte-Foy, Les Presses de l’Université Laval, 2001), 174.

6. Nous reprenons ici le titre de l'ouvrage de C. Lévy-Vroelant, Logements de passage : formes, normes, expériences (Paris, L’Harmattan, 2000), 297.

7. Liste nominative du recensement de 1901, Québec Est, sous-district Saint-Roch, division $4: 7,42-7,48$.

8. Liste nominative du recensement de 1901, Québec Est, sous-district Saint-Sauveur, division $7: 1,47-2,02$. 
deux employés civils qui sont hébergés dans la maison de pension tenue par la veuve Philomène Lambert et ses quatre filles dans le quartier Saint-Louis 9 . Comme bien d'autres habitants de Québec, Anna, Adélard, Odilon, Eugène et John logent chez des familles ou dans des maisons de pension. Ces personnes sont définies dans le recensement comme des pensionnaires, c'està-dire qu'elles paient pension à un particulier ou à un établissement de pension pour que leur soient fournis nourriture et logement.

Les historiens de la famille ont été parmi les premiers à évoquer la présence de pensionnaires dans les villes au $\mathrm{xIx}^{\mathrm{e}}$ siècle. Leurs études révèlent clairement que loger des pensionnaires était un moyen pour les ménages de maintenir ou d'améliorer leur niveau de $v^{1} \mathrm{e}^{10}$. Cela permettait en outre aux femmes et aux jeunes filles d'éviter de travailler à l'extérieur du domicile ${ }^{11}$. Héberger des pensionnaires, souligne judicieusement Bettina Bradbury, pouvait correspondre à une stratégie de survie adoptée par les ménages à différents moments du cycle de vie, notamment lorsque les enfants sont en bas âge ou lors d'un veuvage ${ }^{12}$. De plus, il semble que l'augmentation de la présence des pensionnaires au sein des ménages soit en corrélation avec la diminution du nombre de personnes apparentées qui s'y joignent ${ }^{13}$. Par ricochet, leur présence a pu avoir une incidence sur les formes que prennent les ménages ${ }^{14}$. À divers moments, et dans divers

9. Liste nominative du recensement de 1901, Québec Est, sous-district Saint-Louis, division 3 : $17,17-17,29$.

10. Sherry Olson, «Le peuplement de Montréal», dans Serge Courville, dir., Population et territoire (Sainte-Foy, Les Presses de l'Université Laval, 1996), 65-80; R. O’Day, The Family and Family Relationships, 1500-1900 : England, France and the United States of America (New York, SaintMartin's Press, 1994).

11. Tamara K. Hareven, Families, History and Social Change (Boulder, Westview Press, 2000), 93-94; Collectif Clio, L'histoire des femmes au Québec depuis quatre siècles (Montréal, Le Jour éditeur, 1992), 173; B. Bradbury, "Gender at Work at Home : Family Decisions, the Labour Market, and Girls' Contribution to the Family Economy", dans Bettina Bradbury, dir., Canadian Family History : Selected Readings (Toronto, Copp Clark Pitman, 1992), 177-198.

12. B. Bradbury, Familles ouvrières à Montréal : âge, genre et survie quotidienne pendant la phase d'industrialisation (Montréal, Boréal, 1995), 232-237, 263-264.

13. M. Anderson, Approaches to the History of the Western Family: 1500-1914 (Cambridge, Cambridge University Press, 1995), 12-13 ; B. Bradbury, Familles ouvrières..., op. cit., 85 ; M. B. Katz, The People of Hamilton, Canada West : Family and Class in a Mid-Nineteenth-Century City (Cambridge, Cambridge University Press, 1975), 244; J. Modell et T. Hareven, «Urbanization and the Malleable Household : An Examination of Boarding and Lodging in American Families", Journal of Marriage and the Family, 35,3 (1973) : 467-492.

14. S. Medjuck, "The Importance of Boarding for the Structure of the Household in Nineteenth-Century : Moncton, New Brunswick, and Hamilton, Canada West », Histoire sociale/Social History, 13,25 (1980) : 207-213. 
lieux dans la seconde moitié du XIx ${ }^{\mathrm{e}}$ siècle, la proportion des ménages auxquels se joindraient des pensionnaires oscillerait entre $10 \%$ et $20 \%$, tandis qu'au cours du cycle familial, entre le tiers et la moitié des ménages en logeraient.

Malgré l'apport incontestable des recherches précitées qui s'attachent plutôt au mode d'hébergement, nous demeurons peu renseignés sur les personnes qui prenaient pension. Bradbury souligne de plus que des recherches seraient nécessaires pour mieux connaître ces familles où se trouvaient les pensionnaires. L'organisation résidentielle et financière de cette entente, ajoute-t-elle, tout comme leur intégration au sein des ménages restent des thèmes peu abordés ${ }^{15}$. Dès lors, nous pouvons nous interroger sur l'identité de ces pensionnaires. Qui sont-ils? Forment-ils un groupe homogène?

Une première impression nous porterait à croire qu'ils sont essentiellement de jeunes hommes célibataires, actifs sur le marché du travail et étrangers à la ville qu'ils habitent. Le phénomène des pensionnaires dans les villes nord-américaines en période industrielle s'expliquerait ainsi par la venue de jeunes hommes migrants en recherche de travail et d'un logement ${ }^{16}$. Cependant, plusieurs indices nous poussent à nuancer cette première impression. Le premier de ces indices est que le nombre de femmes à migrer vers la ville est tout aussi appréciable ${ }^{17}$. La féminisation de la main-d'œuvre expliquerait la présence numériquement importante des femmes prenant pension. Elles seraient peut-être de plus en plus nombreuses à préférer ce mode d'hébergement au détriment des formes plus conventionnelles, à savoir l'hébergement au sein de la famille immédiate

15. B. Bradbury, "The Home as a Workplace», dans P. Craven, dir., Labouring Lives : Work and Workers in Nineteenth-Century Ontario (Toronto, University of Toronto, 1995), 454.

16. Par exemple : J. Bullen, «Hidden Workers : Child Labour and the Family Economy in Late Nineteenth-Century Urban Ontario", Labour/Le travail, 18 (1986) : 174 ; J. Modell et T. Hareven, op. cit.; L. A. Glasco, "Migration and Adjustment in the Nineteenth-Century City : Occupation, Properties and Household Structure of Native-Born Whites, Buffalo, New-York, 1855 ", dans T. K. Hareven et M. A. Vinovskis, dir., Family and Population in Nineteenth-Century America (Princeton, Princeton University Press, 1978), 154-178.

17. Citons seulement le cas de Montréal où, déjà en 1861, on aura noté une répartition "égale» des migrants selon le sexe. F. Gagnon, "Parenté et migration : le cas des Canadiens français à Montréal entre 1845-1875 ", Historical Papers of the Canadian Historical Association (1988) : 72 ; en 1901, même si la province affiche un rapport de masculinité de 100,5, la population des villes de 20000 habitants voit ce taux baisser à 89,8. Danielle Gauvreau, "Rats des villes et rats des champs" : populations urbaines et populations rurales du Québec au recensement de 1901 ", Cahiers québécois de démographie, 30,2 (2001) : 176. 
ou étendue, ou encore au sein de la famille de l'employeur. Nous pouvons aussi souligner la présence de familles vivant en pension. Précisons encore que parmi ces familles ont pu se trouver des couples récemment mariés ${ }^{18}$.

Si le migrant - jeune et de sexe masculin surtout - est le protagoniste dans l'histoire des pensionnaires, il convient de ne pas oublier ceux qui s'écartent de ce modèle. C'est justement parce que nous ignorons qui sont ces autres personnes hébergées à Québec qu'il est difficile d'imaginer le rôle que les pensionnaires ont pu jouer dans le développement urbain de la région. Ainsi, nous nous interrogeons sur les rapports que les pensionnaires ont pu établir, d'abord avec les membres des ménages où ils logeaient, mais aussi avec les habitants du voisinage, du quartier et de la ville. Selon nous, une analyse minutieuse de la présence de ces derniers permettrait de mieux cerner les dynamiques urbaines et familiales qui ont donné à Québec son caractère singulier.

L'objectif immédiat de cette note n'est pas de répondre précisément à ces questions. Elle vise plutôt à cerner les caractéristiques de cette population. Ainsi, nous tenterons de savoir qui sont les pensionnaires ayant résidé dans la ville de Québec au tournant du $\mathrm{xx}^{\mathrm{e}}$ siècle. Il importe de noter que nous ne cherchons pas à construire une image type du pensionnaire. C'est en insistant sur les écarts que nous tenterons de mettre à jour la complexité de ce statut.

\section{MÉTHODOLOGIE}

Afin de mieux cerner la population des pensionnaires, l'utilisation des données de recensement nous apparaît pertinente. Cependant, l'exploitation du recensement pour l'étude des formes d'hébergement temporaire reste problématique. Comme opération officielle, le recensement de la population limite notre compréhension des caractéristiques des pensionnaires, car est seule présente la face externe et publique de la vie des personnes étudiées. Sans nier le caractère imparfait et statique de cette source, nous l'utiliserons pour accéder aux pensionnaires. Nous tenterons ainsi de jouer avec les irrégularités produites par ce recensement.

Une exploitation prudente des données du recensement - comme première source d'accès aux pensionnaires - aide à dépasser l'information fragmentaire qu'il nous donne. Le recensement de 1901, plus riche et plus

18. Peter Gossage, Families in Transition : Industry and Population in Nineteenth-Century SaintHyacinthe (Montréal, McGill's-Queen's University Press, 1999), 122-124. 
précis que les précédents, est un outil qui nous permet de mieux cerner la population des pensionnaires ${ }^{19}$.

L’analyse ici faite repose sur le traitement des données nominatives du recensement canadien de 1901. Pour chaque personne, une trentaine d'informations ont été inscrites par les agents recenseurs sur les feuilles que l'on nomme Tableau n ${ }^{\circ}$ 1-Population. Sur ces feuilles de recensement, les individus sont regroupés en fonction du ménage auquel ils appartiennent et de la demeure dans laquelle ils habitent ${ }^{20}$. Dans le cadre du projet de recherche auquel nous participons, les fiches nominatives de recensement ont été saisies ${ }^{21}$. Cette compilation permet d'obtenir des informations pour chacun des 68840 habitants recensés dans la ville en 1901.

Dans le recensement de 1901, apparaît une question destinée à établir la relation de chaque individu avec le chef de famille, du ménage ou de l'institution où il habite. L'ajout de cette question présente l'avantage de fournir des informations sur les liens qui unissent les membres du ménage à son chef. La directive donnée aux recenseurs concernant la variable relation de parenté ou autre avec le chef de ménage se lit comme suit : «Dans la colonne 6 le chef de ménage de chaque famille ou ménage sera inscrit comme tel, et tous les autres selon le degré de parenté, tel épouse, fils, fille, servante, pensionnaire, locataire, associé, etc. ${ }^{22}$.» Comme nous l'avons indiqué par ailleurs, cette nouvelle variable n'est pas sans poser de problème puisque aucune définition du chef n'est explicitement donnée, et ce, jusqu'au recensement de $1951^{23}$. Aussi, la variable relation avec le

19. Nous insistons sur ce point : il sera nécessaire, dans nos recherches ultérieures, de confronter et de jumeler les données du recensement à celles d'autres sources telles que les actes de baptême, de mariage et de décès ainsi que les annuaires de ville, les journaux et les archives notariales.

20. Il semblerait plus approprié de prendre la catégorie «famille ou ménage» pour étudier le logement en milieu urbain québécois. Gilles Lauzon, "Cohabitation et déménagements en milieu ouvrier montréalais : essai de réinterprétation à partir du cas du village de Saint-Augustin ", Revue d'histoire de l'Amérique française, 46,1 (été 1992) : 115-142. Pour plus de détails sur l'usage des catégories famille, ménage et demeure dans les recensements canadiens, voir P. Gossage, op. cit., 196-201; Valérie Laflamme, Familles et modes de résidence en milieu urbain québécois en période industrielle : le cas de la ville de Québec, 1901, mémoire de maîtrise, Université Laval, 2000, 41-48.

21. Cette base de données fut construite dans le cadre du projet Transitions démographiques : le cas de la ville de Québec, 1871-1901, codirigé par Marc St-Hilaire et Richard Marcoux. Voir aussi C. Fleury, Rapport sur la construction de la base de données relative au recensement de 1901 (Université Laval, ronéo., 1998) www.phsvq.cieq.ulaval.ca/Bases.

22. Ministère de l'Agriculture du Canada, Quatrième recensement du Canada : 1901, 1 : Population (Ottawa, S. E. Dawson, 1902), xviii.

23. R. Marcoux et J. Mongeau, "Votre ménage a-t-il changé de "boss" entre 1971 et 1981 ?», Cahiers québécois de démographie, 18,1 (1989) : 118. Sur les difficultés que pose la variable relation avec le chef de ménage dans le recensement canadien de 1901, voir aussi G. Darroch, 
chef de ménage est insuffisante pour identifier toutes les relations familiales pouvant exister au sein du ménage ${ }^{24}$.

À la lecture des fiches nominatives du recensement, il est aisé de savoir qui sont les pensionnaires puisqu'ils sont explicitement désignés comme tels par le chef de ménage. Nous nous intéressons ici aux 3091 personnes déclarées pensionnaires. Cette sous-population représente $4,5 \%$ de la population totale recensée en 1901 dans la ville de Québec.

\section{PRINCIPALES CARACTÉRISTIQUES DES PENSIONNAIRES DE QUÉBEC}

Rappelons pour commencer que notre objectif immédiat consiste à mieux savoir qui sont les pensionnaires, et non où ils sont hébergés. Ultérieurement, nous reprendrons la distinction souvent faite entre les pensionnaires habitant au sein de ménages particuliers, en maisons de pension et en hôtels ${ }^{25}$. Précisons dès maintenant que $74 \%$ des pensionnaires logent chez des particuliers où se trouvent moins de 5 pensionnaires, et que $26 \%$ vivent en maison de pension ou à l'hôtel.

\section{Sexe, âge et statut matrimonial}

À Québec en 1901, 51,6\% des 3091 pensionnaires sont de sexe masculin. Ces données sont différentes de celles obtenues dans les villes où l'on avait déjà remarqué la présence de pensionnaires. Ailleurs au Canada et à d'autres moments, les femmes pensionnaires seraient moins présentes. Par exemple, à Hamilton en 1851, à Moncton en 1851 et 1861, et à Sudbury en 1901, les

"Constructing Census Families and Classifying Households : "Relationship to Head of Family Household" in the 1901 Census of Canada ", Historical Methods, 33,4 (2000) : 206-210; V. Laflamme, op. cit., 48-52. Sur l'aspect normatif des questions sur la famille et le ménage dans les recensements : D. S. Smith, "The Meanings of Family and Households : Change and Continuity in the Mirror of the American Census ", Population and Development Review, 18,3 (1992) : 421-456; B. Bradbury, «Single Parenthood in the Past : Canadian Census Categories, 1891-1951, and the "Normal Family" ", Historical Methods, 33,4 (2000) : 211-217.

24. Entre autres, les liens unissant les pensionnaires sont occultés du fait que leur relation est donnée en fonction du chef. À titre d'exemple, une analyse préliminaire indique que 17,6\% des pensionnaires partagent le même patronyme qu'au moins un autre des pensionnaires résidant au sein du même ménage. Vraisemblablement, ce sont surtout des frères et sœurs. Voir S. Ruggles, «Family Interrelationships», Historical Methods, 28 (1995) : 52-58.

25. Cette distinction est pour le moins importante dans la mesure où elle recoupe des réalités différentes. La pension chez les particuliers équivaudrait à un "substitut» de la famille quittée, alors que la pension en maison de pension et en hôtel se ferait dans un contexte où se développerait une «sous-culture urbaine». M. Peel, «On the Margins : Lodgers and Boarding in Boston, 1860-1900», Journal of American History, 72,4 (1986) : 813-834 ; R. F. Harney, «Boarding and Belonging», Urban History Review, 2 (1978) : 8-37. 
femmes formeraient respectivement $29 \%, 19,3 \%, 16,7 \%$ et $5 \%$ de l'effectif des pensionnaires ${ }^{26}$. Cette forte proportion de femmes pensionnaires peut être spécifique au contexte économique et social de la ville de Québec.

Regardons d'un peu plus près la structure d'âge des pensionnaires, afin d'évaluer si elle varie selon le $\operatorname{sex}^{27}$ (graphique 1). De manière générale, les hommes pensionnaires sont jeunes : le quart des pensionnaires de sexe masculin se trouvant à Québec au début du siècle ont de 20 à 24 ans. Les femmes pensionnaires, malgré une légère hausse à l'âge de 20 à 29 ans, sont réparties beaucoup plus équitablement que les hommes. Plus de la moitié des pensionnaires masculins ont entre 15 et 29 ans $(53,9 \%)$, et le tiers seulement des femmes ont ces âges (38,0\%). En proportion, les femmes pensionnaires sont aussi plus nombreuses que les hommes lorsqu'elles ont 35 ans et plus.

Si être pensionnaire est synonyme de jeunesse, il semble qu'à Québec il soit aussi synonyme de vieillesse. La présence de pensionnaires âgés varie cependant selon le sexe. Les femmes pensionnaires âgées de 65 à 89 ans sont aussi nombreuses que celles âgées de 25 à 29 ans $^{28}$. Par ailleurs, les hommes pensionnaires de 65 ans et plus représentent $6,5 \%$ de l'effectif, ce qui est faible.

Hommes comme femmes, les pensionnaires sont célibataires trois fois sur quatre ${ }^{29}$. Parmi les pensionnaires mariés, seuls trois sur quatre logent avec leurs conjoints ${ }^{30}$. Les causes de séparation peuvent être diverses :

26. M. B. Katz, op. cit.; S. Medjuck, «Family and Household Composition in NineteenthCentury : The Case of Moncton New-Brunswick, 1851-1871», Canadian Journal of Sociology, 4,3 (1979) : 275-286; A. Prud'Homme, «Les pensionnaires apparentés et non apparentés dans la région de Sudbury, 1891-1901 ", dans L. Rousseau et Y. Gauthier, dir., Actes de la sixième journée du savoir de l'acfas (Sudbury, Institut franco-ontarien, 2000), 187-217. Ailleurs qu'au Canada, la tendance à ce que les pensionnaires soient majoritairement des hommes est confirmée par des auteurs tels que : M. Anderson, Family Structure in Nineteenth-Century Lancashire (Cambridge, Cambridge University Press, 1978), 230; L. A. Glasco, op. cit.

27. Nous avons exclu de cette analyse 5 pensionnaires pour lesquels l'âge en années n'est pas donné. Sont donc ici analysés 3086 pensionnaires, dont 1592 sont de sexe masculin et 1494 de sexe féminin.

28. Au total, $13,4 \%$ des femmes pensionnaires ont 65 ans et plus, alors que $12,3 \%$ d'entre elles sont âgées de 25 à 29 ans.

29. Dans une proportion de $73,7 \%$ les femmes sont célibataires, 9,5\% sont mariées, $16,7 \%$ sont veuves et $0,1 \%$ sont divorcées. Quant aux hommes, $78,8 \%$ sont célibataires, $10,7 \%$ sont mariés, $10,2 \%$ sont veufs et $0,4 \%$ sont divorcés.

30. Pour 211 pensionnaires mariés, 164 sont recensés avec leurs époux. Ce qui signifie qu’aux yeux du recensement, 56 femmes et 96 hommes mariés sont «séparés» de leurs conjoints. Ces résultats ne donnent qu'un aperçu du phénomène de la séparation des conjoints puisqu'il est possible "que plusieurs couples séparés de fait» figurent comme cohabitant dans les données du recensement. B. Bradbury, op. cit., 245. 
GRAP H I Q U E 1

Répartition des pensionnaires de la ville de Québec selon le sexe et l'âge, 1901

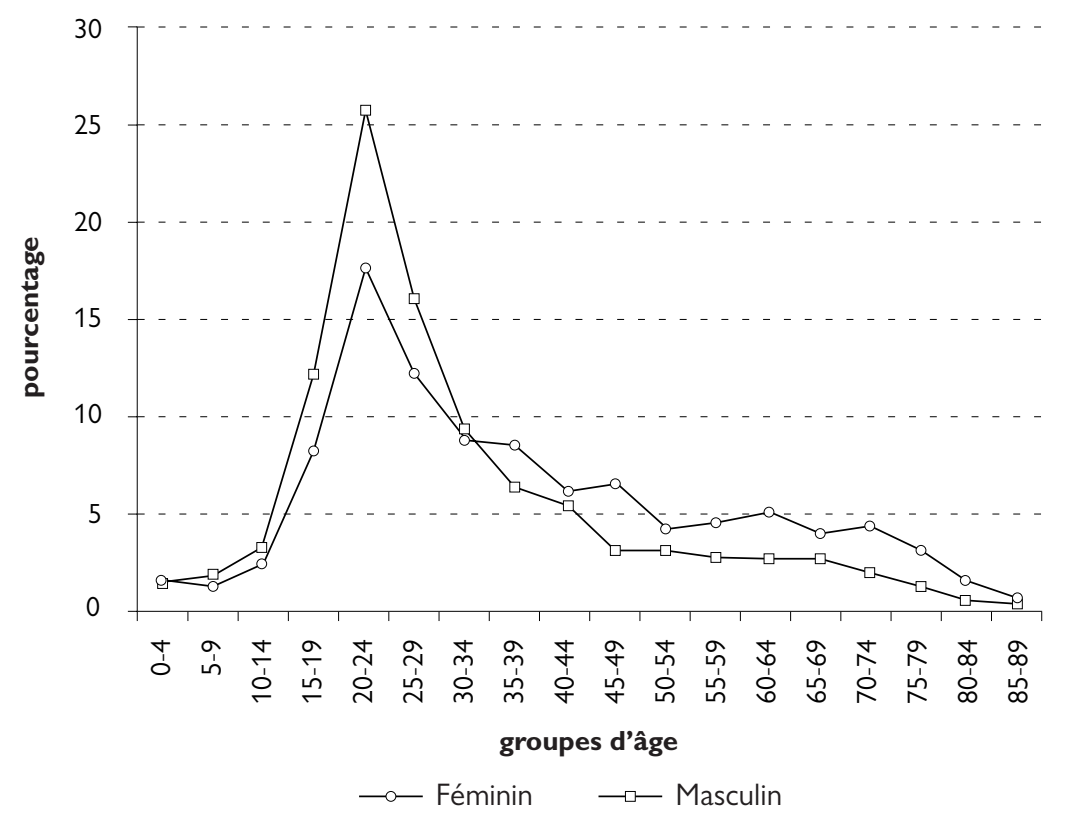

départ d'un des conjoints - souvent l'homme - pour travailler, concubinage, séparation de fait et autres.

Les jeunes couples et les jeunes familles prennent aussi pension : parmi les couples mariés identifiés, $47,6 \%$ ont moins de 30 ans, et $35,4 \%$ ont des enfants âgés de moins de 5 ans.

Nous retrouvons aussi une proportion importante de veuves. Si les veufs et veuves sont souvent âgés, il importe de noter que les hommes sont plus jeunes que les femmes. Cela laisse entrevoir le caractère plus temporaire de la prise de pension des hommes au moment d'un veuvage, et celui plus permanent pour les veuves ${ }^{31}$.

Grâce à ces résultats, nous voyons qu'être pensionnaire est une pratique sexuée et très variable selon l'âge : hommes et femmes prennent pension à différents moments de leur vie. Le cas des femmes veuves est éclairant

31. Au total, $62,4 \%$ des pensionnaires veuves sont âgées de 55 à 79 ans, alors que pour les hommes cette proportion n'est que de 49,2\%. À l'inverse, $23,3 \%$ des veuves et 37,2\% des veufs ont de 30 à 54 ans. 
sur ce point. Ce qui laisse même présager qu'être pensionnaire puisse être plus qu'une étape intermédiaire et transitoire servant à intégrer le milieu urbain. Elle pourrait être une manière d'habiter la ville.

Nous avons observé que les femmes pensionnaires sont aussi nombreuses que les hommes. Cela permet de supposer que la prise de pension a pu être délaissée par les hommes au tournant du siècle ${ }^{32}$. Les raisons de ce recul, s'il existe, demeurent inconnues. Au même moment, la prise de pension a pu être recherchée par les jeunes femmes en quête d'autonomie. Elles préféraient peut-être établir des rapports plus formels avec leurs hôtes, ce qui restait plus délicat au sein de leur parentèle ${ }^{33}$. Avant de conclure à une mutation du sens et de la fonction de la pension, plusieurs éléments demandent vérification. Notamment, il reste à savoir quels sont les parcours géographiques, sociaux et professionnels, la durée des séjours des pensionnaires ainsi que les rapports entretenus avec les membres des ménages qui les logent ${ }^{34}$.

\section{Lieu de naissance}

Comme nous l'avons précisé plus haut, la ville de Québec a été un foyer d'appel pour les habitants des régions rurales avoisinantes. Pour mesurer la présence de ruraux dans la population des pensionnaires, nous pouvons exploiter les variables relatives à leurs lieux de naissance ${ }^{35}$.

On note d'abord la forte présence d'une population née dans la province de Québec ${ }^{36}$. Ce qui confirme d'une autre manière qu'au tournant du $x^{\mathrm{e}}$ siècle, la ville de Québec est de moins en moins un lieu d'accueil pour les migrants internationaux.

L'utilisation de la variable lieu de naissance nécessite certaines précautions ${ }^{37}$. D’abord, la présence d'un pensionnaire à Québec originaire d'une

32. Voir à ce sujet l'excellente étude du déclin de la pratique d'hébergement de pensionnaires à Toronto de Harris. R. Harris, "The End Justified the Means : Boarding and Rooming in a City of Homes, 1890-1951 », Journal of Social History, 26,2 (1992) : 331-358.

33. T. K. Hareven, op. cit., 94.

34. Détail intéressant à noter : Lucia Ferretti observe qu'entre les pensionnaires et les membres de la famille d'accueil «des liens se tissent qui conduisent plus d'une fois à l'Autel». Lucia Ferretti, "Mariage et cadre de vie familiale dans une paroisse ouvrière montréalaise : Sainte-Brigide, 1900-1914", Revue d'histoire de l'Amérique française, 39,2 (automne 1985) : 246.

35. Les organisateurs du recensement demandaient de préciser le pays ou la province ainsi que la région (rurale ou urbaine) de naissance.

36. Au total, 93,0\% des pensionnaires sont originaires du Québec, loin derrière suivent les personnes nées aux États-Unis et en Angleterre qui représentent quant à elles 2,52\% des pensionnaires. Quatorze personnes pour lesquelles le lieu de naissance n'a pas été donné sont ici exclues. 
zone rurale ne nous informe ni sur le moment de son arrivée en ville ni sur son aspect définitif. Un va-et-vient entre le lieu de naissance et le lieu d'accueil n'est pas inconcevable. La simple utilisation de cette variable ne nous permet pas de retracer le parcours des habitants de Québec. Ultérieurement, nous retracerons - par les actes de baptême, de mariage et de décès - les parcours des pensionnaires de Québec. En second lieu, nous ignorons comment sont définies les régions "rurales » et «urbaines» par les organisateurs du recensement, par les recenseurs et par les recensés. Des lieux comme Montréal, Québec, Trois-Rivières, Saint-Hyacinthe peuvent, sans ambiguïté, être définis comme étant des régions urbaines. Mais que dire des autres régions?

Une faible majorité $(52,2 \%)$ des pensionnaires nés dans la province sont issus des milieux ruraux : pour les hommes cette proportion est de 55,0\%, contre $49,7 \%$ pour les femmes. C'est dans la tranche d'âge des 15 à 29 ans que les hommes nés en milieu rural sont les plus présents (tableau 1). Cet écart est encore plus remarquable pour les 20 à 24 ans. Ces jeunes hommes sont alors deux fois plus nombreux que ceux nés en milieu urbain. Chez les pensionnaires âgés de 30 à 49 ans, la tendance s'inverse faiblement : les hommes pensionnaires nés en milieu urbain sont légèrement plus présents que les ruraux. Si les effectifs sont suffisamment forts pour en déduire que les jeunes hommes pensionnaires sont surtout nés en milieu rural, il faut se garder de conclure trop rapidement l'inverse pour les pensionnaires de plus de 30 ans, en raison de la faiblesse de l'écart et des effectifs. La répartition par âge des femmes pensionnaires selon leur lieu de naissance présente peu d'écarts. Il y a presque toujours autant de femmes pensionnaires venant des zones rurales que des zones urbaines. À elle seule, la variable lieu de naissance n'est pas suffisante pour étudier la différence des profils des pensionnaires. Il nous manque malheureusement la date de l'arrivée des pensionnaires à Québec. Prendre pension dans la ville de Québec ne s'effectue pas dans les mêmes circonstances pour un jeune homme célibataire nouvellement arrivé à Québec que pour une femme veuve originaire de cette même ville. Néanmoins, les jeunes pensionnaires habitant Québec en 1901 sont en majorité originaires des milieux ruraux. De plus, l'analyse de l'origine des pensionnaires par le lieu de naissance renforce l'hypothèse que les pensionnaires n'appartiennent pas exclusivement à la population migrante. 
TAB LEAU 1

Âge, sexe et lieu de naissance (rural ou urbain) des pensionnaires, Québec 1901

\begin{tabular}{c|c|c|c|c}
\hline \multirow{2}{*}{$\begin{array}{c}\text { Nombres } \\
\text { absolus }\end{array}$} & \multicolumn{2}{|c|}{ Féminin } & \multicolumn{2}{c}{ Masculin } \\
\cline { 2 - 5 } & Rural (\%) & Urbain (\%) & Rural (\%) & Urbain (\%) \\
\hline $0-4$ & 0,29 & 2,89 & 0,12 & 2,87 \\
$5-9$ & 0,58 & 1,88 & 0,62 & 3,93 \\
$10-14$ & 1,46 & 2,60 & 2,97 & 3,93 \\
$15-19$ & 8,60 & 8,24 & 12,87 & 11,80 \\
$20-24$ & 20,41 & 15,46 & 33,17 & 17,70 \\
$25-29$ & 11,37 & 13,15 & 16,58 & 16,49 \\
$30-34$ & 8,60 & 8,82 & 7,80 & 11,20 \\
$35-39$ & 8,75 & 8,53 & 4,58 & 7,56 \\
$40-44$ & 5,83 & 6,36 & 4,70 & 6,51 \\
$45-49$ & 6,85 & 6,65 & 1,98 & 4,39 \\
$50-54$ & 3,50 & 4,91 & 2,97 & 2,72 \\
$55-59$ & 4,52 & 4,48 & 2,23 & 2,42 \\
$60-64$ & 6,27 & 4,19 & 1,86 & 3,18 \\
$65-69$ & 4,23 & 3,61 & 2,97 & 2,57 \\
$70-74$ & 3,64 & 4,62 & 2,60 & 0,91 \\
$75-79$ & 3,06 & 2,46 & 1,11 & 1,21 \\
$80-84$ & 1,60 & 0,72 & 0,50 & 0,61 \\
$85-89$ & 0,44 & 0,43 & 0,37 & 0,00 \\
\hline Total (\%) & 100,00 & 100,00 & 100,00 & 100,00 \\
\hline Total (N) & 686 & 692 & 808 & 661 \\
\hline & & & & \\
\hline & & & & \\
\hline
\end{tabular}

Source : Exploitation des données du recensement de 1901 (PHSVQ/CIEQ).

\section{Origine et religion}

Les villes québécoises de la fin du $\mathrm{XIX}^{\mathrm{e}}$ et du début du $\mathrm{xx}^{\mathrm{e}}$ siècle ont été habitées par trois communautés ethnoreligieuses principales : à savoir, les catholiques d'origine française, les catholiques d'origine irlandaise et les anglophones non catholiques, pour la plupart de confession protestante ${ }^{38}$.

38. M. St-Hilaire et R. Marcoux, op. cit.; S. Olson, «Le peuplement... », op. cit. ; S. Olson, et P. Thornton, «La croissance naturelle des Montréalais au $\mathrm{XIX}^{\mathrm{e}}$ siècle», Cahiers québécois de démographie, 30,2 (2001) : 191-230. 
Il semble qu'à ces communautés étaient associés des valeurs et des modes de vie spécifiques, et qu'elles ont vécu dans des conditions économiques distinctes. À Montréal notamment, on observe une surreprésentation des Canadiens français dans les métiers d'ouvriers, de journaliers et de travailleurs non spécialisés ${ }^{39}$. À l'inverse, les protestants sont plus équitablement répartis et occupent en plus forte proportion des métiers de marchands ou de commis.

Dans une ville comme Québec, cette division en trois grandes communautés ethnoreligieuses s'est surtout manifestée par une segmentation sociale et géographique. Bien entendu, entre ces communautés, il n'existait pas de barrières infranchissables. Il n'en demeure pas moins que l'appartenance à une communauté ou à une autre a pu avoir un effet, quoique faible, sur les comportements résidentiels. C'est du moins ce qu'une analyse des structures des ménages a laissé entrevoir ${ }^{40}$.

Pour savoir si l'appartenance à l'une des communautés ethnoreligieuses a une incidence sur le fait de prendre pension, nous avons classé les pensionnaires selon cette variable ${ }^{41}$. La distribution des pensionnaires en fonction de la variable ethnoreligieuse doit en outre être opposée à la répartition totale des habitants de Québec selon ce même critère (tableau 2). Cela permettra de dégager le caractère ethnique et religieux des pensionnaires.

De cette mise en parallèle ressort une grande similitude des proportions. Sachant que Québec était un foyer d'appel pour les ruraux des régions avoisinantes, qui sont, rappelons-le, principalement d'origine française et de religion catholique, nous aurions pensé observer une plus forte présence des pensionnaires catholiques français dans la ville. Or, ce n'est pas le cas. Les arrivants catholiques français ont-ils préféré vivre en famille plutôt qu'en pension? Nous ne pouvons, dans le cadre de cette note, explorer cette piste. Elle est pour le moins intéressante, dans la mesure où elle permettrait de mettre en lumière les phénomènes de migration en chaîne et de réseaux familiaux ${ }^{42}$.

39. S. Olson, op. cit., 85-88; B. Ramirez, Crossing the $44^{\text {th }} \ldots$, op. cit., 123.

40. V. Laflamme, «Familles et modes de résidence en milieu urbain québécois au début du $x^{\mathrm{e}}$ siècle : le cas de la ville de Québec, 1901 ", Cahiers québécois de démographie, 30,2 (2001) : 261-288.

41. Nous nous sommes servis des informations fournies dans le recensement sur l'origine et la religion.

42. De tels processus ont été observés par des auteurs comme B. Ramirez, On the Move, FrenchCanadian and Italian Migrants in the North Atlantic Economy, 1860-1914 (Toronto, McClelland \& Stewart, 1991); Gérard Bouchard, Quelques arpents d'Amérique : population, économie, famille au Saguenay, 1838-1971 (Montréal, Boréal, 1996); S. Olson, “"Pour se créer un avenir”. Stratégies de 
TA B LEA U 2

Pensionnaires et non-pensionnaires selon l'appartenance aux principales communautés ethnoreligieuses, Québec 1901

\begin{tabular}{l|c|c|c|c}
\hline \multirow{2}{*}{$\begin{array}{c}\text { Communauté } \\
\text { ethnoreligieuse }\end{array}$} & \multicolumn{2}{|c|}{ Pensionnaires } & \multicolumn{2}{c}{ Population totale* } \\
\cline { 2 - 5 } & $\%$ & $\mathbf{N}$ & $\%$ & $\mathbf{N}$ \\
\hline Catholique française & 79,97 & 2472 & 82,65 & 51034 \\
Irlandaise catholique & 6,50 & 201 & 7,51 & 4635 \\
Anglaise non catholique & 6,86 & 212 & 4,58 & 2829 \\
Autres & 6,66 & 206 & 5,26 & 3246 \\
\hline Total & 100,00 & 3091 & 100,00 & 61744 \\
\hline
\end{tabular}

* Excluant les pensionnaires et les ménages institutionnels.

Source : Exploitation des données du recensement de 190I (PHSVQ/CIEQ).

L'affinité ethnoreligieuse entre les pensionnaires et les familles qui les hébergent demande à être approfondie. Lees a trouvé qu'à Londres les familles irlandaises récemment installées étaient plus enclines à héberger des membres de la famille éloignée et des pensionnaires que les habitants de la ville installés depuis plus longtemps. Les nouveaux arrivants, laisse supposer Lees, hébergeaient des pensionnaires non seulement pour augmenter leurs revenus, mais aussi pour fournir un accueil chaleureux aux nouveaux venus dans un environnement souvent hostile, et où les logements se faisaient rares ${ }^{43}$. Étudier cette question dans une ville comme Québec où la population s'est presque entièrement renouvelée permettrait de lever le voile sur les modes d'intégration à la vie urbaine. Par ricochet, nous serions alors en mesure de mieux connaître les modes de «recrutement» des pensionnaires. Nous arriverions ainsi à saisir davantage les liens unissant les pensionnaires et les familles.

\section{Statut d'emploi et profession}

Une meilleure connaissance des pensionnaires de Québec passe par une analyse des statuts socioprofessionnels. Le recensement de 1901 a l'avantage de

couples montréalais au $\mathrm{xIx}^{\mathrm{e}}$ siècle", Revue d'histoire de l'Amérique française, 51,3 (hiver 1998) : 357389 ; F. Gagnon, op. cit.; J.-C. Robert, «Urbanisation et population : le cas de Montréal en 1861 », Revue d'histoire de l'Amérique française, 35,4 (mars 1982) : 523-535.

43. L. H. Lees, "Patterns of Lower-Class Life : Irish Slum Communities in NineteenthCentury London", dans S. Thernstrom et R. Sennett, dir., Nineteenth-Century Cities : Essays in the New Urban History (New Haven, Yale University Press, 1968), 359-385. 
nous fournir ces renseignements qui peuvent être analysés selon la grille de classification d'Erikson et Goldthorpe, communément appelée EGP ${ }^{44}$.

Comme pour l'analyse de la variable ethnoreligieuse, nous confronterons la sous-population des pensionnaires à la population totale. Nous retiendrons la population âgée de 15 à 59 ans seulement, qui correspond à la population active. Une remarque s'impose : notre analyse ne peut prendre en compte que les professions déclarées aux recenseurs. Le recensement a tendance à occulter ou à sous-évaluer le travail féminin ${ }^{45}$ ainsi que la "pluriactivitée ${ }^{46}$ ".

La répartition des hommes et des femmes selon les statuts d'emploi diverge (tableau 3). Dans le cas des pensionnaires, ces divergences s'atténuent. Les hommes pensionnaires, tout comme la population masculine de Québec, occupent le statut d'employé trois fois sur quatre. Ils sont moins souvent à leur compte et patrons que les autres hommes de Québec (7,6\% contre $14,36 \%)$.

Une femme pensionnaire sur deux est employée, ce qui contraste nettement avec les 22,0\% d'employées de la population féminine de Québec. On peut aussi relever que ces dernières sont moins à leur compte et moins rentières que les femmes pensionnaires. Enfin, beaucoup plus de femmes pensionnaires déclarent avoir un statut d'emploi (43,6\% contre 74,5\%).

Le tableau 4 nous apporte quelques éléments de compréhension supplémentaires $^{47}$. Nous voyons que presque un tiers $(30,7 \%)$ des pension-

44. À la classification EGP, M. De Sève et S. Desrosiers proposent une adaptation canadienne que nous retenons ici. M. De Sève et S. Desrosiers, Les classes d'Erikson, Goldthorpe et Portecarero : une adaptation canadienne, communication présentée au $62^{\mathrm{e}}$ Congrès de l'ACFAS (Montréal, ronéo., mai 1994), 2-25. Voir aussi C. Fleury, «Les classes d'Erikson, Golthorpe et Portecarero (EGP) : rapport technique sur l'utilisation et l'intégration des codes EGP dans le recensement de 1851, 1871 et 1901 " (Université Laval, ronéo., 2000) www.phsvq.cieq.ulaval.ca/Bases ; R. Erikson et J. Goldthorpe, The Constant Flux: A Study of Class Mobility in Industrial Societies (Oxford, Clarendon Press, 1992), 430. Sur les questions relatives aux conditions de travail et aux revenus des salariés dans le recensement de 1901, voir P. Baskerville, «Displaying the Working Class: The 1901 Census of Canada ", Historical Methods, 33,4 (2000) : 229-234; E. W. Sager, "The National Sample of the 1901 Census of Canada: A New Source for the Study of the Working Class», web.uvic.ca/hrd/cfp/esshap.htm.

45. Sur l'économie familiale et l'invisibilité du travail féminin et domestique, voir notamment : B. Bradbury, Familles..., op. cit., chapitres 5 et 6 ; Collectif Clio, op. cit.

46. Les directives données aux recenseurs sont explicites sur ce point : «La première ou principale occupation est la seule qui devra être inscrite; c'est-à-dire celle dont dépend principalement la condition de vie et dont sont principalement dérivés les gages, émoluments ou revenus. » Ministère de l'Agriculture du Canada, Quatrième recensement du Canada: 1901, 1 : Population (Ottawa, S. E. Dawson, 1902), xix.

47. Sont ici exclues les personnes âgées de 15 à 59 ans ne déclarant pas une profession : 89 hommes et 497 femmes pensionnaires, et 1255 hommes et 14735 femmes de la population totale. 
TABLEAU 3 Statut d'emploi des pensionnaires et non-pensionnaires
de 15 à 59 ans selon le sexe, Québec 1901

\begin{tabular}{|c|c|c|c|c|c|c|c|c|}
\hline \multirow{3}{*}{$\begin{array}{c}\text { Statut } \\
\text { d'emploi }\end{array}$} & \multicolumn{4}{|c|}{ Pensionnaires } & \multicolumn{4}{|c|}{ Population totale* } \\
\hline & \multicolumn{2}{|c|}{ Masculin } & \multicolumn{2}{|c|}{ Féminin } & \multicolumn{2}{|c|}{ Masculin } & \multicolumn{2}{|c|}{ Féminin } \\
\hline & $\%$ & $\mathbf{N}$ & $\%$ & $\mathbf{N}$ & $\%$ & $\mathbf{N}$ & $\%$ & $\mathbf{N}$ \\
\hline À son compte & 3,60 & 49 & 3,75 & 42 & 6,18 & 996 & 1,88 & 376 \\
\hline Employé & 73,53 & 1000 & 48,13 & 539 & 72,91 & 11741 & 21,85 & 4365 \\
\hline Patron & 4,04 & 55 & 1,25 & 14 & 8,18 & 1317 & 0,83 & 165 \\
\hline Rentier & 1,18 & 16 & 3,21 & 36 & 0,55 & 88 & 0,94 & 188 \\
\hline Aucun statut & 17,57 & 239 & 43,66 & 489 & 12,13 & 1953 & 74,49 & 14881 \\
\hline Autres & 0,07 & 1 & 0,00 & 0 & 0,06 & 9 & 0,01 & 2 \\
\hline Total & 100,00 & 1360 & 100,00 & 1120 & 100,00 & 16104 & 100,00 & 19977 \\
\hline
\end{tabular}

* Excluant les pensionnaires.

Source : Exploitation des données du recensement de 1901 (PHSVQ/CIEQ).

naires occupent des emplois de bureau, de service et non manuels (classes I-II, et IIIab), alors qu'un cinquième $(21,6 \%)$ seulement des hommes de Québec se trouve dans cette catégorie. À l'inverse, les pensionnaires occupent moins fréquemment les emplois à caractère manuel (classes IV-VI, VIIa, VIIb). Ces tendances s'inversent pour les femmes : les pensionnaires de sexe féminin sont plus nombreuses dans les métiers manuels et moins nombreuses dans les emplois de bureau, de service et non manuels que les autres femmes de Québec. Notons enfin la relative équivalence de la structure socioprofessionnelle des hommes et des femmes pensionnaires.

La présence des pensionnaires dans toutes les catégories socioprofessionnelles nous permet d'affirmer que le logement en pension ne résulte pas d'une situation socioprofessionnelle particulière. Toute proportion gardée, les pensionnaires (hommes comme femmes) n'occupent pas plus les métiers semi ou non qualifiés que la population totale. De toute évidence, les pensionnaires - les hommes surtout — ne vivent pas en situation de précarité ${ }^{48}$.

La question des salaires des pensionnaires apporterait d'utiles précisons. On peut dès à présent noter que le salaire des femmes, moindre que celui

48. Puisque presque une femme sur deux ne déclare pas de profession, il serait précipité de tirer les mêmes conclusions. 
TABLEAU 4

Catégories socioprofessionnelles des pensionnaires et des non-pensionnaires de 15 à 59 ans déclarant une profession, selon le sexe, Québec 1901

\begin{tabular}{|c|c|c|c|c|c|c|c|c|}
\hline \multirow{3}{*}{$\begin{array}{l}\text { Catégories } \\
\text { socio- } \\
\text { professionnel }\end{array}$} & \multicolumn{4}{|c|}{ Pensionnaires } & \multicolumn{4}{|c|}{ Population totale* } \\
\hline & \multicolumn{2}{|c|}{ Masculin } & \multicolumn{2}{|c|}{ Féminin } & \multicolumn{2}{|c|}{ Masculin } & \multicolumn{2}{|c|}{ Féminin } \\
\hline & $\%$ & $\mathbf{N}$ & $\%$ & $\mathbf{N}$ & $\%$ & $\mathbf{N}$ & $\%$ & $\mathbf{N}$ \\
\hline $\begin{array}{l}\text { Classe de } \\
\text { service } \\
\text { supérieure et } \\
\text { inférieure (I-II) }\end{array}$ & 9,13 & 116 & 6,42 & 40 & 6,64 & 986 & 3,07 & 161 \\
\hline $\begin{array}{l}\text { Classe des } \\
\text { petits employés } \\
\text { non manuels } \\
\text { (IIllab) }\end{array}$ & 21,56 & 274 & 22,31 & 139 & 14,99 & 2226 & 35,60 & 1866 \\
\hline $\begin{array}{l}\text { Sous-classe } \\
\text { des petits } \\
\text { entrepreneurs } \\
\text { (IVab) }\end{array}$ & 5,51 & 70 & 8,99 & 56 & 12,86 & 1910 & 9,56 & 501 \\
\hline $\begin{array}{l}\text { Sous-classe des } \\
\text { entrepreneurs } \\
\text { secteur } \\
\text { primaire }(\mathrm{IV} c)\end{array}$ & 0,16 & 2 & 0,00 & 0 & 0,61 & 90 & 0,13 & 7 \\
\hline $\begin{array}{l}\text { Contremaîtres, } \\
\text { techniciens } \\
\text { inférieurs et } \\
\text { travailleurs } \\
\text { manuels } \\
\text { qualifiés }(\mathrm{V}-\mathrm{VI})\end{array}$ & 29,11 & 370 & 38,68 & 241 & 35,96 & 5340 & 29,55 & 1549 \\
\hline $\begin{array}{l}\text { Sous-classe } \\
\text { des travailleurs } \\
\text { manuels semi } \\
\text { et non qualifiés } \\
\text { (Vlla) }\end{array}$ & 16,84 & 214 & 15,89 & 99 & 19,25 & 2858 & 13,41 & 703 \\
\hline $\begin{array}{l}\text { Sous-classe } \\
\text { des travailleurs } \\
\text { manuels } \\
\text { secteur } \\
\text { primaire (VIIb) }\end{array}$ & 0,71 & 9 & 0,00 & 0 & 0,23 & 34 & 0,04 & 2 \\
\hline Indéterminé & 16,99 & 216 & 7,70 & 48 & 9,46 & 1405 & 8,64 & 453 \\
\hline Total & 100,00 & 1271 & 100,00 & 623 & 100,00 & 14849 & 100,00 & 5242 \\
\hline
\end{tabular}

* Excluant les pensionnaires.

Source : Exploitation des données du recensement de 190I (PHSVQ/CIEQ). 
des hommes, limitait certainement leur choix de résidence ${ }^{49}$. On peut envisager que parmi celles-ci se trouvaient de nombreuses célibataires. Ce célibat s'accompagnait de l'exercice de professions ou de métiers de clercs, d'institutrices, de domestiques non résidentes, de secrétaires, d'ouvrières et de vendeuses. Ces emplois devaient être suffisamment rémunérateurs pour les faire vivre, mais insuffisants pour qu'elles habitent seules ${ }^{50}$. C'est sans doute le cas, par exemple, de Régine Beaulieu, couturière célibataire de 45 ans qui loge chez Charles et Marie Huot ${ }^{51}$ ou de Mary Milke, institutrice célibataire de 35 ans prenant pension chez Émilie Talbot et ses quatre filles ${ }^{52}$.

À la lecture de ces données, plusieurs questions se posent. Que font les pensionnaires ne déclarant pas de profession? Car près d'une femme sur deux $(44,4 \%)$ et moins d'un homme sur dix $(6,5 \%)$ recensés en tant que pensionnaires n'ont pas déclaré de profession. Pour s'acquitter de leur pension, les pensionnaires ont-ils d'autres ressources? On peut imaginer que certains d'entre eux ont passé des ententes particulières avec leurs logeurs. Plusieurs de ces femmes pensionnaires «sans profession " participaient sans doute au travail domestique en échange de quoi elles étaient hébergées (garde d'enfants, ménage, petits travaux d'entretien, couture, etc.).

\section{CONCLUSION}

Nous avons effectué ici une première analyse des caractéristiques de la population des pensionnaires. Des tendances ont été mises au jour qui témoignent surtout de l'hétérogénéité de cette population. Elle n'est pas constituée uniquement de jeunes hommes célibataires. Les femmes en représentent la moitié. Cependant, hommes et femmes ne sont pas pensionnaires aux mêmes âges. Les pensionnaires de sexe masculin ont plutôt de 15 à 29 ans, tandis que les femmes se répartissent plus équitablement à

49. Pour une analyse des corrélations entre le salaire des femmes, le coût de la pension et le lieu de résidence, voir G. Cross et P. Shergold, «The Family Economy and the Market: Wages and Residence of Pennsylvania Women in the 1890's ", Journal of Family History, 11,3 (1986) : 245266.

50. Voir aussi, S. Cross, «La majorité oubliée : le rôle des femmes à Montréal au XIX ${ }^{\mathrm{e}}$ siècle», dans Marie Lavigne et Yolande Pinard, dir., Les femmes dans la société québécoise (Montréal, Boréal Express, 1977), 33-59; C. Strange, Toronto's Girl Problem: The Perils and Pleasures of the City, 18801930 (Toronto, University of Toronto Press, 1995).

51. Liste nominative du recensement de 1901, Québec Est, sous-district Jacques-Cartier, division 3: 6,12-6,14.

52. Liste nominative du recensement de 1901, Québec Centre, sous-district Du Palais, division $3: 3,11-3,17$. 
tous les âges. L'étude des lieux de naissance des pensionnaires a montré que neuf pensionnaires sur dix étaient nés dans la province du Québec. Ils sont à parts égales originaires des régions rurales et urbaines. De plus, il n’y a pas de surreprésentation d'une communauté ethnoreligieuse particulière même dans une ville majoritairement francophone et catholique. Enfin, l'hétérogénéité apparaît aussi dans la répartition des pensionnaires selon les catégories socioprofessionnelles. À ce propos, rien n'indique que les pensionnaires hommes vivent en situation précaire. Il semblerait même qu'ils occupent plutôt des emplois de bureau. Le cas des femmes reste plus incertain, dans la mesure où une femme sur deux ne déclare pas sa situation socioprofessionnelle.

Ces premières analyses précisent les pistes de recherche qui demandent à être explorées. D’abord, il nous semble nécessaire d'étudier les affinités unissant les pensionnaires aux membres des ménages qui les logent. En effet, comment connaitre les conditions dans lesquelles vivent les pensionnaires sans les situer dans leur environnement? Cela permettrait de décrire les réseaux familiaux et de voisinage qui organisent la ville - objet de recherche encore peu exploré au Québec ${ }^{53}$. Il reste aussi à préciser les quartiers de la ville où résident de préférence les pensionnaires ainsi que les raisons qui les poussent à prendre pension dans un contexte immobilier précis.

Cependant, le recensement comme source exclusive pour étudier ces caractéristiques de la prise de pension nous impose des limites. En effet, le recensement n'est pas suffisant pour envisager les dynamiques d'intégration à l'urbain (spatiales et sociales) dans une perspective inscrite dans la durée et multidimensionnelle. L'exploitation d'autres sources, tels les actes de baptême, de mariage, de décès, les actes notariaux, les annuaires de ville et les journaux, viendrait enrichir la compréhension des dynamiques urbaines et familiales de la ville de Québec au tournant du $\mathrm{xx}^{\mathrm{e}}$ siècle. Nous cherchons ainsi à mettre au jour l'existence d'une multitude de systèmes qui interagissent dans une constante transformation de la ville et de sa population.

53. C. Dessureault, «Parenté et stratification sociale dans une paroisse rurale de la vallée du Saint-Laurent au milieu du XIX ${ }^{e}$ siècle", Revue d'histoire de l'Amérique française, 53,3 (hiver 2001): 411-447. 